\title{
Random walks, diffusion limited aggregation in a wedge, and average conformal maps
}

\author{
Leonard M. Sander ${ }^{a)}$ \\ Michigan Center for Theoretical Physics, Department of Physics, University of Michigan, Ann Arbor, \\ Michigan 48109-1120 \\ Ellák Somfaib) \\ Instituut-Lorentz, University of Leiden, P. O. Box 9506, 2300 RA Leiden, Netherlands
}

(Received 22 November 2004; accepted 2 February 2005; published online 17 June 2005)

\begin{abstract}
We investigate diffusion-limited aggregation (DLA) in a wedge geometry. Arneodo and collaborators have suggested that the ensemble average of DLA cluster density should be close to the noise-free selected Saffman-Taylor finger. We show that a different, but related, ensemble average, that of the conformal maps associated with random clusters, yields a nontrivial shape which is also not far from the Saffman-Taylor finger. However, we have previously demonstrated that the same average of DLA in a channel geometry is not the Saffman-Taylor finger. This casts doubt on the idea that the average of noisy diffusion-limited growth is governed by a simple transcription of noise-free results. (C) 2005 American Institute of Physics. [DOI: 10.1063/1.1876932]
\end{abstract}

The diffusion-limited aggregation model uses aggregating random walkers to form a random fractal pattern. We can also use random walkers to generate a conformal map from the exterior of the cluster to the exterior of the unit circle. The map contains all the information about the growth probabilities for various points on the cluster. We define the pattern generated by the ensemble average of the conformal maps as a kind of mean-field pattern for DLA. In previous work we showed that there seemed to be an intriguing relationship between DLA in a channel and the noise-free Saffman-Taylor finger. We show here that this relationship is much more ambiguous when we apply it to DLA in a wedge geometry. This result casts doubt on the "averaging conjecture" which holds that the average of noisy growth "remembers" noise-free results.

\section{INTRODUCTION}

The last century has abounded with examples of unexpected richness in the problem of brownian motion as formulated by Einstein in his seminal paper in $1905 .^{1}$ In the same year, Pearson ${ }^{2}$ pictured the process as a random walk. In the past century random walks and brownian motion have become central themes of statistical physics. The problem is particularly astonishing in that it constantly generates new ways to think about nature, and new descriptions of physical processes. One example was the discovery by Witten and Sander ${ }^{3,4}$ that aggregating random walkers give rise to random fractal patterns. This process, diffusion-limited aggregation (DLA), is simple to formulate: a seed particle is put at a point, and then a random walking particle is launched and allowed to proceed until it touches the seed; then it stops. Then another walker diffuses until it touches one of the first two, and so on. We study the cluster generated. This is the

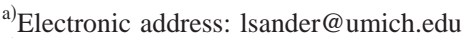

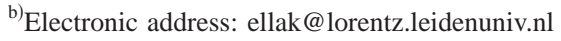

simplest paradigm of noise-dominated growth limited by diffusion, a common natural process.

This process has been studied intensively over the last 23 years (for a review, see Ref. 5), but there is still not complete theoretical understanding, though some recent progress has been made ${ }^{6}$ in describing local correlations. In this paper we investigate a property of the ensemble of DLA clusters namely the generation of average shapes. Our motivation comes from the remarkable suggestion of Arneodo and collaborators $^{7,8}$ that the (somewhat arbitrarily defined) ensemble average shape of noisy DLA clusters would be the pattern generated by noise-free diffusion-limited growth. We will refer to this as the averaging conjecture. The relevant case of noise-free growth is the Saffman-Taylor viscous finger, ${ }^{9,10}$ i.e., the shape of the surface of an inviscid fluid invading a viscous one, as water into oil. The work of Arneodo et al. showed that Saffman-Taylor fingers in a channel and a wedge were close to, but not exactly the same, as DLA averages. However, the work had a number of arbitrary parameters and, in the wedge, there were serious ambiguities.

We investigated the averaging conjecture in our work ${ }^{11}$ on DLA in a channel with reflecting boundaries at the walls. There, we formulated a new definition of the average shape by averaging the conformal map ${ }^{12-14}$ that generates the cluster-this amounts to weighting points on the surface according to their growth probability. In that work we found that the average shape of DLA (using our definition) was not a Saffman-Taylor viscous finger.

However, a number of authors ${ }^{6,15}$ had already questioned whether DLA and viscous fingers are actually closely related growth processes; DLA does not have surface tension like viscous fingering, but rather a fixed particle size that defines the tip radius. We followed up the suggestion ${ }^{6,16}$ that fluid flow with surface tension is closely related to a variant of DLA called the dielectric breakdown model (DBM) ${ }^{17}$ with parameter $\eta \approx 1.2$ (to be defined below). In fact, the suitably 
averaged DBM clusters then turned out to fit the SaffmanTaylor shape quite closely.

Here we look at the related problem of DLA in a wedge. Previously ${ }^{18}$ we have shown that tip-splitting in this geometry gives us access to local correlations. In the present context of looking at average shapes, the wedge is interesting in several respects. This is a richer problem than that of the channel in that the wedge angle is a free parameter, and the shape of the fluid invasion is more complex. ${ }^{19-22}$ We pose the question of whether the DBM shape in a wedge is also a good approximation to the Saffman-Taylor finger in a wedge.

This study has a special significance in this focus issue on brownian motion. Our work here is primarily numerical. The simulations are all done by random walker sampling: we generate DLA clusters, DBM clusters, and conformal maps by this single method which, as it happens, is by far the most efficient method available. This century-old technique has not lost its freshness and power.

\section{DLA AND DBM IN A WEDGE}

There are now available very sophisticated schemes for generating DLA clusters. The one we use is based on the method of hierarchical maps. ${ }^{23}$ In this method space is divided into regions of various sizes which help keep track of the nearest points on the cluster. Then the random walker can make large jumps in empty regions, vastly speeding up the computation. With this method the time to create an $N$ particle cluster is proportional to $N^{p}$ with $p \approx 1.1$.

We need to make DLA clusters in a wedge with reflecting boundary conditions. We do this by means of a trick. If there is a wall at some position, every time we deposit a walker, we deposit an image walker reflected in the wall. Suppose we are interested in $90^{\circ}$ wedges. Then we have two perpendicular walls, and four walkers are deposited at once. Using this method we can use a radial DLA code to produce wedges of opening angles $180^{\circ} / n, n=1,2, \ldots$. In this paper we will concentrate on $90^{\circ}$ and $60^{\circ}$ wedges. An example of a $90^{\circ}$ wedge is shown in Fig. 1.

In the following we will see that we need to consider DBM clusters. These are defined as follows: we imagine that the cluster is a grounded conductor with unit charge. Outside the cluster define a potential, $\phi$, so that

$$
\nabla^{2} \phi=0, \quad \phi_{s}=0 .
$$

This defines an "electric field" on the surface, $\partial \phi / \partial n_{s}$. Then the dielectric breakdown model takes the growth velocity on the surface of the cluster to be

$$
v_{n} \propto\left[\frac{\partial \phi}{\partial n}\right]_{s}^{\eta} .
$$

We interpret this equation probabilistically: $v_{n}$ is taken as the density of the growth probability on the surface, $\mu$. In practice, add a particle at a point on the cluster with probability proportional to $v_{n}$. It is known that at large scales DBM clusters with $\eta=1$ have the same scaling as DLA.

The original method ${ }^{17}$ to grow DBM clusters was to solve Eq. (1) by relaxation. This algorithm is very slow and is not practical for generating large clusters. Recently ${ }^{24}$ we

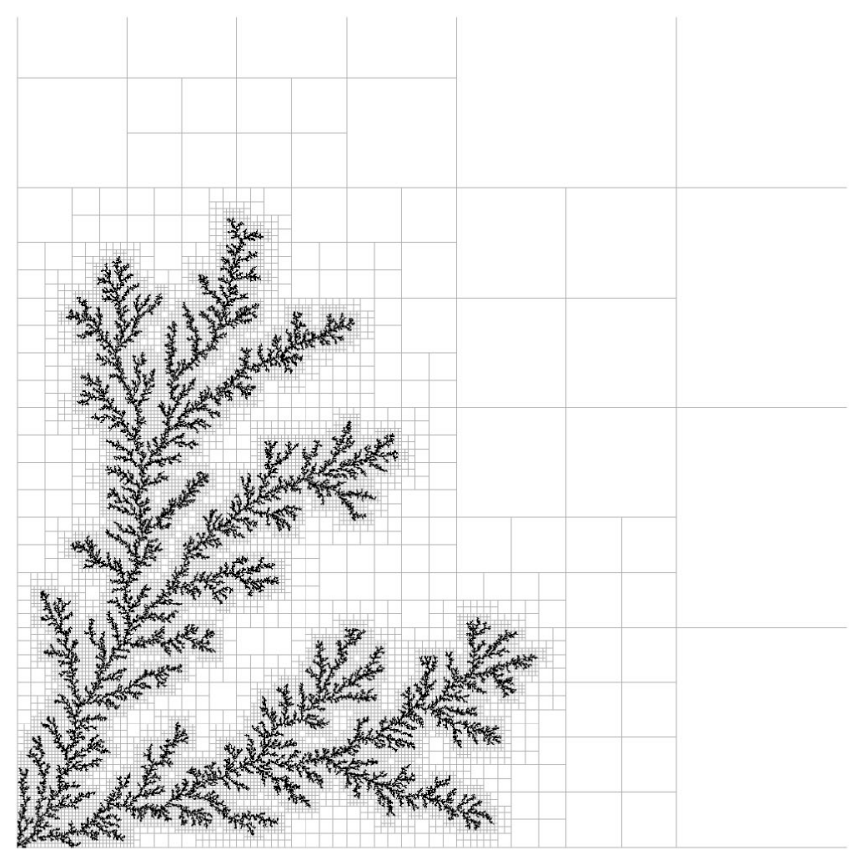

FIG. 1. A DLA cluster in a $90^{\circ}$ wedge with reflecting boundaries. The structure of the hierarchical maps is also shown.

have introduced a method of growing DBM clusters by random walker sampling. The key to this method is to define the age, $a_{1}$, of a growth site. This is the number of random walkers that have landed anywhere on the cluster since the last particle grew at the site. We can also define $a_{k}$, the number of walkers that have landed since the $k$ th most recent particle grew there.

Since the frequency of landing of random walkers is proportional to $\mu$, it is clear that $1 / a_{1}, 2 / a_{2}, \ldots$ at a site are estimates of $\mu$ at that site. We have shown ${ }^{24}$ that this estimate is adequate to allow us to grow DBM clusters. In our work here we use $a_{3}$ to estimate the probability.

The method of growth is as follows: if a particle lands at a site with low probability, we arrange to have it add little to the cluster and, at high probability sites, add a good deal. This is accomplished by adding a mass, $\delta m=A \mu^{\eta-1} \propto a_{n}^{1-\eta}$. (The power is $\eta-1$ because we already have a probability $\mu$ for the walker to land at the site.) In practice, when a particle is added at a site, it is moved onto the existing particle so that a portion proportional to $a_{k}^{1-\eta}$ contributes to new growth. We also change the prefactor, $A$, as we go along to make an efficient code. For details, see Ref. 24. Examples of this are shown in Fig. 2.

The computations we discuss below are averages over an ensemble of off-lattice DLA and DBM clusters grown in this way. Our DLA clusters had 1000000 particles in the wedge and we averaged over an ensemble of 400 realizations. For the DBM clusters we had 500000 particles in the $60^{\circ}$ wedge, 1000000 particles in the $90^{\circ}$ wedge, and also 400 realizations. Our motivation for going to these large sizes is the fact that DLA suffers slow crossovers. ${ }^{14,25}$ If we use small clusters, we are not seeing the asymptotic behavior. 

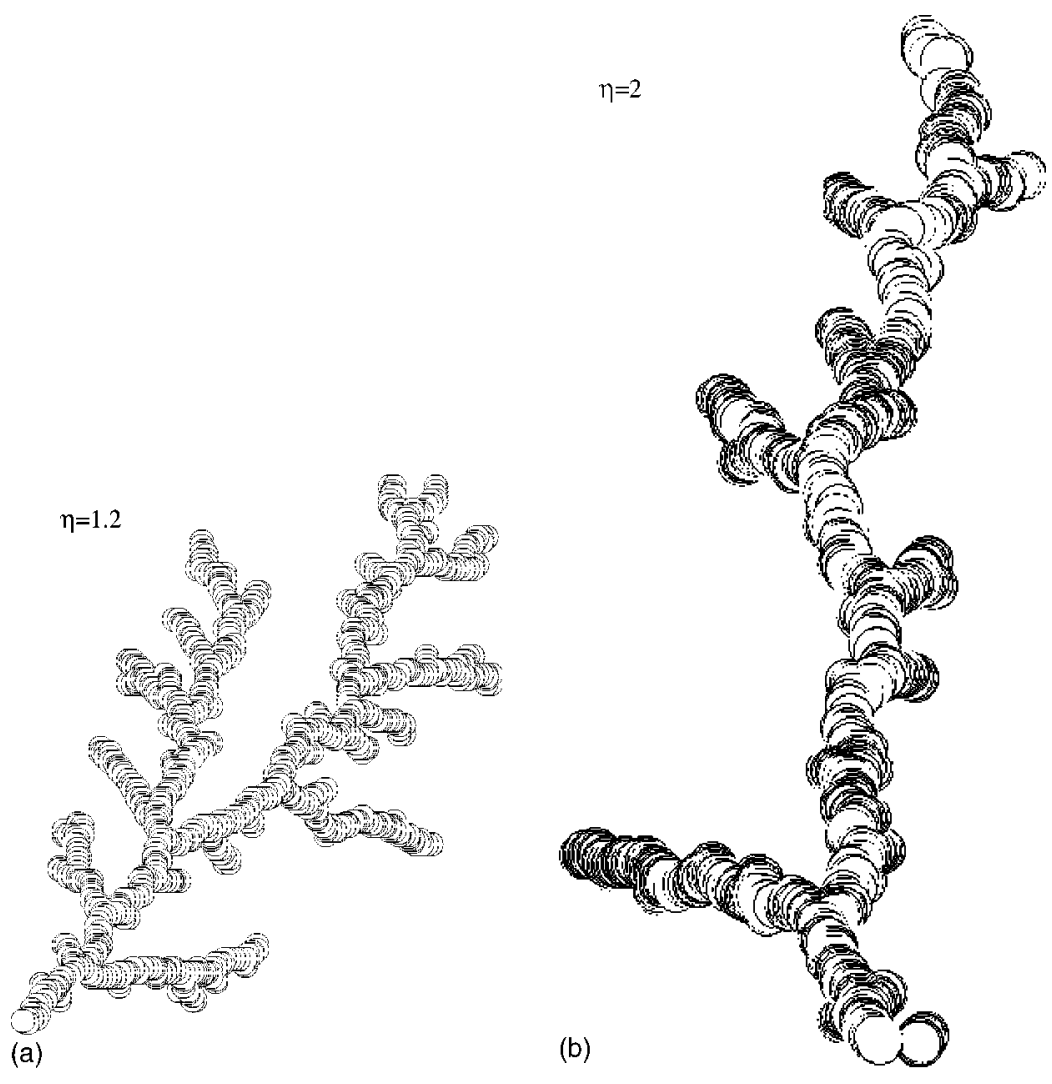

FIG. 2. A portion of DBM clusters for two different values of $\eta$. For $\eta=2$ the overlapping of particles and the enhanced growth at the tips is easy to see.

\section{CONFORMAL MAPS}

In recent years a number of groups ${ }^{12,13}$ have looked at DLA in an entirely new way. The cluster shape is considered to be a grounded conductor, as above, and the complex potential, $\phi$, is sought, as in Eq. (1). The technique introduced was to define a conformal map from the exterior of the cluster to the exterior of the unit circle. The Laplace equation, Eq. (1), can be solved easily outside the circle, and the solution mapped back to the cluster. Of course, the solution for $\partial \phi / \partial n$ is uniform on the circle. Thus the image of two parts of the cluster perimeter with the same growth probability will map to parts of the unit circle with the same length. Put another way, the inverse images of uniformly spaced points on the unit circle are distributed on the cluster with density $\mu$.

In order to construct the map Hastings and Levitov ${ }^{12}$ invented an iterative technique which grows a cluster and calculates the map at the same time. This is a practical method, but slow. In Ref. 14 we constructed an alternative method which is much faster. We grow a cluster by the conventional fast scheme using random walkers. Then we freeze the cluster at the desired size, launch $n$ random walkers as probes, and record where they land. Then the values of the map on the unit circle are found as follows. We choose one of landing positions as a starting point, say along the $x$ axis, and the number the walkers around the perimeter of the cluster. By the observation above, if we are at walker $m$, we know that we have turned an angle $\theta=2 \pi m / n+\mathcal{O}\left(n^{-1 / 2}\right)$ from the image of the $x$ axis on the unit circle. In Ref. 14 we used this information, which samples the boundary values of the conformal map, to construct the map itself by analytic continuation.

For our purposes here we use the information in a simpler way. We constructed the map to the unit circle using $n$ $=100000$ for each cluster that we grew. For each member of the ensemble there is a point, $\mathbf{r}(\theta)$ whose image is a point on the unit circle at $e^{i \theta}$. Our definition of the ensemble average shape generated by the DLA or DBM process ${ }^{24}$ is the ensemble average of $\mathbf{r}(\theta)$, i.e., the centroid of those points (see Fig. 3). We sample points on the unit circle with sufficient resolution to define the average shape. Since points at the leading tips of the clusters grow with high probability, they would be oversampled if we chose points on the unit circle

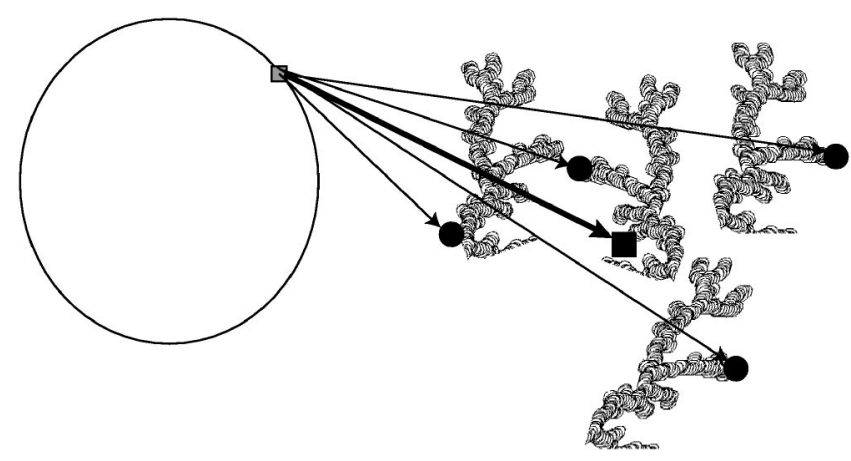

FIG. 3. Schematic of the averaging procedure. For different realizations of the cluster, the same point on the unit circle (gray square) is mapped to different points in space on the different clusters that make up the ensemble. Our definition of the ensemble average map points to the geometric center of those points (bold arrow). 


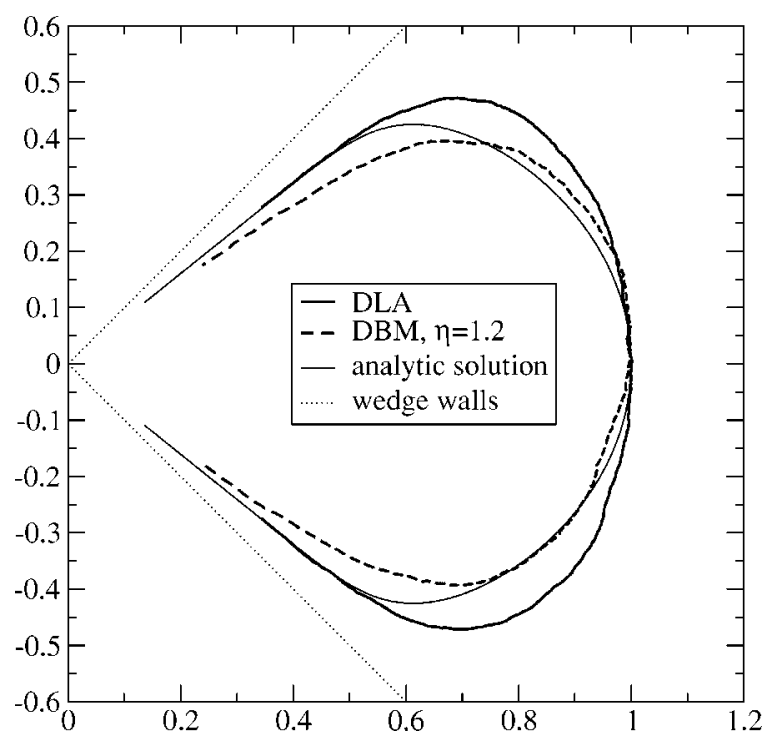

FIG. 4. The averaged profile of DLA and DBM clusters with $\eta=1.2$ in a $90^{\circ}$ wedge. Also shown is the analytic solution for a Saffman-Taylor finger with the selected $\lambda$ from Refs. 19 and 20.

uniformly. Instead, we use an adaptive procedure until the average shape is well defined. In Figs. 4 and 5 we show the average shapes in $90^{\circ}$ and $60^{\circ}$ wedges.

This definition has a number of advantages: it is unambiguous, in contrast to definitions based on density profiles (see below). It is an average which is weighted by probability, that is, we are sampling where the growing tips of the cluster are located. It might be accessible to theoretical investigation since the growth process is the defining property of DLA.

\section{THE AVERAGING CONJECTURE AND MEAN-FIELD THEORY}

\section{A. Saffman-Taylor fingers}

The investigation of Hele-Shaw flow in a channel is an old subject and a good deal is known about it. In particular,

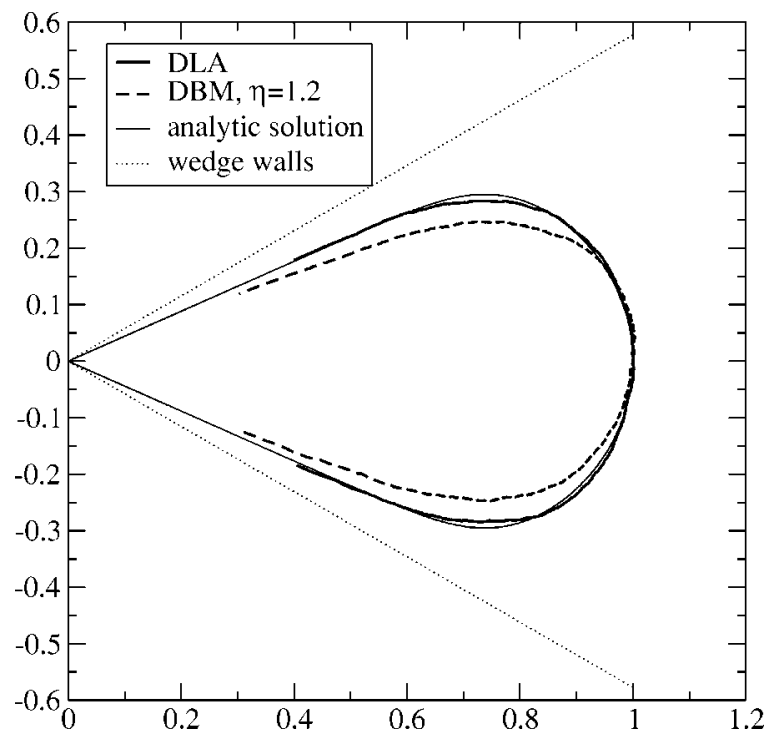

FIG. 5. The averaged profile of DLA and DBM clusters with $\eta=1.2$ in a $60^{\circ}$ wedge. Also shown is the analytic solution for a Saffman-Taylor finger with the selected $\lambda$ from Refs. 19 and 20. the invasion of a viscous fluid by an inviscid one is one of the most famous of all pattern formation problems. ${ }^{9,10}$ The general result is that the inviscid fluid forms a finger, the Saffman-Taylor finger, which fills a fraction, $\lambda$, of the channel. For each $\lambda$ in $[0,1]$ there is a solution to the fluid-flow equations without surface tension. For small surface tension, the pattern is very close to that with zero surface tension and $\lambda=\frac{1}{2}$. This is called the selected value. The finger elongates in time and is of constant shape in a moving reference frame.

The theory of this sort of flow is remarkably well developed. A few basic notions are necessary here: the fluid velocity, $\mathbf{v}$, is derivable from a potential in this case and is governed by D'Arcy's law. The upshot of these two facts is that

$$
\nabla \cdot \mathbf{v}=0=\nabla^{2} \phi ; \quad v_{n} \propto\left[\frac{\partial \phi}{\partial n}\right]_{s} .
$$

That is, fluid flow obeys the same equations as DBM [cf. Eq. (2)] with $\eta=1,{ }^{26}$ so that the Saffman-Taylor finger has a good deal in common with DLA. The bubble of inviscid fluid plays the role of the aggregate. However, there are two salient differences: DLA does not have surface tension but, rather, a finite size cutoff, and DLA is dominated by noise, whereas the Saffman-Taylor finger is a stable, noise-free pattern that is observed for slow flows in a channel.

There is another problem related to the channel problem which also admits an exact solution, that of viscous fingering in a wedge. In this case, for short times there is also a selected shape in experiments, at least for a finite time. ${ }^{19} \mathrm{Tu}$ and Ben Amar ${ }^{20-22}$ worked out the theory in this case and showed that there is a self-similar shape that is selected. It is a nontrivial pattern whose form is given by a differential equation that needs to be solved numerically. Associated with the problem is a selected angle, defined as the opening angle at the base of the wedge (see Figs. 4 and 5). The ratio of the opening angle of the finger to that of the wedge is also called $\lambda$. With nosurface tension there is a solution for all $\lambda$, but, once more, there is a selected value which depends on the wedge angle. There is a complication in the fluid-flow problem. If the inviscid fluid is pumped at a constant pressure, as time goes on there will always come a point when the pattern is unstable against tip-splitting.

\section{B. Averaging}

Arneodo and collaborators ${ }^{7,8}$ exploited the resemblance between DLA growth and viscous fingering in the following way: they speculated that the average of many DLA clusters would, in some sense, remove the noise and recover the noise-free pattern. Since DLA has no surface tension, but rather a fixed particle size (playing, roughly, the role of the capillary length), they assumed that the limit of small surface tension was the appropriate one. This is what we refer to as the averaging conjecture.

They tested the conjecture by generating on-lattice DLA clusters in a channel and averaging the density, point by point. ${ }^{7}$ The density average, $\rho(\mathbf{r})$, is a function that goes to zero at the edge of the channel and has a maximum, $\rho_{\max }$, at 
the center. One of the level sets of this function, $\mathbf{r}_{\mathbf{s}}:\left\{\rho\left(\mathbf{r}_{\mathbf{s}}\right)\right.$ $\left.=\frac{1}{2} \rho_{\max }\right\}$ traced out a Saffman-Taylor finger with $\lambda=\frac{1}{2}$.

However, closer scrutiny made the picture more complex. Lattice effects are known to distort DLA clusters and are irrelevant to the kind of physics being considered. Therefore, they returned to the problem ${ }^{8}$ and generated off-lattice DLA clusters. They found that the level set at $\frac{1}{2} \rho_{\max }$ filled $56 \%$ of the channel rather than $50 \%$, or, alternatively, the level set that was needed to make $\lambda=\frac{1}{2}$ was at $0.6 \rho_{\max }$.

For the same problem in wedges of various opening angles, on-lattice DLA density averages once more gave remarkable agreement with selected fingers. ${ }^{7}$ However, offlattice the situation was different: tip-splitting made the front of the fingers too flat, though there was qualitative agreement with the opening angle of the average density. And, it was necessary to choose a level set somewhat arbitrarily. In fact, since the overall density decreased as the length of the finger increased, it was necessary to define the opening angle by taking a fraction of the maximum density at that distance from the apex of the wedge.

\section{Mean-field theory}

Inspired by the averaging conjecture, Levine, $\mathrm{Tu}$, and collaborators ${ }^{27,28}$ revised the mean-field theory of Witten and Sander ${ }^{4}$ to attempt to write a proposed set of equations for the mean density of a cluster. They found qualitative agreement with Ref. 7. In Ref. 8 the theory was extended, and other work has followed this up more recently. ${ }^{29}$

The salient results of this theory are that a level set, defined as above, more-or-less fits the channel finger, but that the wedge-based fingers are too flat.

\section{Average conformal maps for DLA in a wedge}

The numerical underpinnings of the averaging conjecture are troublesome in several ways. At the most simple level, the number of particles in the clusters studied was very small, of order $10^{3}-10^{4}$. We have already remarked that DLA in that regime is far from asymptotic. More significantly, there are far too many fitting parameters in the discussion. The level set is chosen arbitrarily, and, for many of the discussions, $\lambda$ is chosen to fit the pattern.

We returned to this problem with our new definition of the ensemble average pattern, described above. In a channel we were able to show ${ }^{11}$ that the average shape does not fit any Saffman-Taylor finger. The finger width of the pattern corresponded to $\lambda \approx 0.6$, just as in Ref. 8 , despite the different definitions of the average. With the resolution that we had available, we were able to show definitively that the finger we generated did not fit the Saffman-Taylor pattern for any $\lambda$.

Here we return to the problem for growth in a wedge. Using the techniques described above, we have grown DLA clusters and averaged for wedge angles of $90^{\circ}$ and $60^{\circ}$. The results are shown in Figs. 4 and 5 along with the analytic solutions for the selected finger shape. Now, quite remarkably, the opening angle of the finger does fit rather well to the selected $\lambda$. Note that there are no adjustable parameters in this fit other than an overall scale factor. The tip of the finger in the $90^{\circ}$ wedge is flatter than it should be. However, for $60^{\circ}$ the fit is reasonably good.

\section{E. Average conformal maps for DBM in a wedge}

In our channel work we tried to salvage the averaging conjecture in the following way. There is a theoretical suggestion $^{6,16}$ that the correct analogy between fluid flow and flow with surface tension was not with DLA. According to this theory, if we consider DBM models with a short scale cutoff rule where the tip radii are limited by $R \gtrsim|\partial \phi / \partial n|^{-m}$, then one can find families of equivalent models in the $(\eta, m)$ parameter space. The equivalence is based on the same behavior of leading tips in the models with different parameters. In this framework the Hele-Shaw flow, where the tip radius obeys the relation $R v^{1 / 2}=$ const, corresponds to $m=\frac{1}{2}$, while for DLA with fixed size particles $m=0$. According to the equivalence, flow with surface tension cutoff $(\eta=1, m$ $=\frac{1}{2}$ ) corresponds to the model with fixed $R$ (i.e., $m=0$ ) but $\eta \approx 1.2$. We were very encouraged in the channel by this analogy because the average of DBM with this $\eta$ did fit a Saffman-Taylor finger with $\lambda=\frac{1}{2}$ rather well.

In the wedge, as Figs. 4 and 5 show, the DBM fingers $d o$ not fit the analytic shape. They tend to be too narrow. This is exactly the opposite of the situation in the channel. Once more, there are no adjustable parameters available to us to fit the shape.

\section{SUMMARY AND DISCUSSION}

When we began this study we were confident that we would be able to put the averaging conjecture on a firm footing, based on our experience with the channel geometry. Our expectations were not at all fulfilled. It is possible to maintain that we have, in fact, eliminated the averaging conjecture altogether. Perhaps this strong conclusion is premature, but, certainly, the situation is not very clear. To summarize: in a channel, averaged DBM using the mapping of Refs. 6 and 16 gives a Saffman-Taylor finger with the correct $\lambda$ but averaged DLA does not. In the wedge, averaged DLA gives the correct finger opening angle for both wedges that we looked at, but averaged DBM does not.

We might be tempted to say that our proposal for averaging based on conformal maps should simply be discarded. If we do that, we are reduced to using density averages which do not fit the analytic fingers any better (worse, in fact) and are ambiguous to boot.

In passing, we should comment on the situation with tip-splitting. We do not agree that DLA averages in a wedge should tip-split for any opening angle $>0$. We base this on our work in the wedge geometry. ${ }^{18}$ In that paper we used not reflecting boundary conditions, but periodic boundaries for the wedge. We looked at angular correlations of the density for DLA clusters and found that for small wedge angles there was a minimum in the correlation function half-way between the branch and its image. We interpreted this by saying that for small angles there was one major branch. For large angles, $\gtrsim 144^{\circ}$ we found a secondary maximum in the correlation function, i.e., more than one major branch. These results do not directly carry over to the present case, but, 
qualitatively, we think that tip-splitting in the sense just described is not at all clearly present in this case. There is a numerical result of Ref. 8 for a $60^{\circ}$ wedge which seems to contradict this, but we are confident that our statistics were much better.

It is likely that tip-splitting for DLA is a probabilistic matter. We suspect that in a $90^{\circ}$ wedge some clusters split, but the majority do not. This could account for the small, but definite, disagreement between the shape of the tip of the averaged clusters in the $90^{\circ}$ wedge with the analytic solution. However, there is almost certainly a qualitative difference between DLA and fluid flow with respect to tip-splitting. Also, the extent of tip-splitting found in mean-field theory 8,28 does not agree with our DLA averages or with the results of Ref. 18

However, the whole question of tip-splitting in this system is controversial. There remains the possibility that our results and those of Ref. 18 are finite-size effects, i.e., that we have not grown large enough clusters at small angles. In a limited effort to see finite size effects, we generated DLA clusters in a $90^{\circ}$ wedge similar to those shown in Fig. 4, but ten times smaller in size. The average profile of the small DLA clusters fell in between the analytical curve and the large DLAs. This points in the direction that in the $90^{\circ}$ wedge larger DLA clusters-deviating more from the analytical curve- have a larger degree of tip splitting. However, future work is necessary to fully settle this issue.

The project of formulating a description of the average over the DLA ensemble still seems to us to be quite a worthy one. However, the present results show that the current state of the art in this area is far from giving the definitive answer.

\section{ACKNOWLEDGMENTS}

We are indebted to Dave Kessler for the suggestion of measuring the average conformal map. We thank the Center for the Study of Complex Systems at the University of Michigan for computing resources. LMS acknowledges par- tial support by NSF Grant No. DMS-0244419. ES would like to thank the University of Michigan for hospitality, and the PHYNECS training network of the European Commission for financial support (Contract No. HPRN-CT-2002-00312).

${ }^{1}$ A. Einstein, Ann. Phys. 17, 549 (1905).

${ }^{2}$ K. Pearson, Nature (London) 72, 294 (1905).

${ }^{3}$ T. A. Witten and L. M. Sander, Phys. Rev. Lett. 47, 1400 (1981).

${ }^{4}$ T. A. Witten and L. M. Sander, Phys. Rev. B 27, 5686 (1983).

${ }^{5}$ L. M. Sander, Contemp. Phys. 41, 203 (2000).

${ }^{6}$ R. C. Ball and E. Somfai, Phys. Rev. Lett. 89, 135503 (2002).

${ }^{7}$ A. Arneodo, Y. Couder, G. Grasseau, V. Hakim, and M. Rabaud, Phys. Rev. Lett. 63, 984 (1989).

${ }^{8}$ A. Arneodo, J. Elezgaray, M. Tabard, and F. Tallet, Phys. Rev. E 53, 6200 (1996).

${ }^{9}$ P. G. Saffman and G. Taylor, Proc. R. Soc. London, Ser. A 245, 312 (1958).

${ }^{10}$ P. Pelce, New Visions on Form and Growth (Oxford U. P., Oxford, 2004).

${ }^{11}$ E. Somfai, R. C. Ball, J. P. DeVita, and L. M. Sander, Phys. Rev. E 68, 020401 (2003).

${ }^{12}$ M. B. Hastings and L. S. Levitov, Physica D 116, 244 (1998).

${ }^{13}$ B. Davidovitch, H. G. E. Hentschel, Z. Olami, I. Procaccia, L. M. Sander, and E. Somfai, Phys. Rev. E 59, 1368 (1999).

${ }^{14}$ E. Somfai, L. M. Sander, and R. C. Ball, Phys. Rev. Lett. 83, 5523 (1999).

${ }^{15}$ F. Barra, B. Davidovitch, A. Levermann, and I. Procaccia, Phys. Rev. Lett. 87, 134501 (2001).

${ }^{16}$ R. C. Ball and E. Somfai, Phys. Rev. E 67, 021401 (2003).

${ }^{17}$ L. Niemeyer, L. Pietronero, and H. J. Wiesmann, Phys. Rev. Lett. 52, 1033 (1984).

${ }^{18}$ D. A. Kessler, Z. Olami, J. Oz, I. Procaccia, E. Somfai, and L. M. Sander, Phys. Rev. E 57, 6913 (1998).

${ }^{19}$ H. Thome, M. Rabaud, V. Hakim, and Y. Couder, Phys. Fluids A 1, 224 (1989).

${ }^{20}$ Y. H. Tu, Phys. Rev. A 44, 1203 (1991).

${ }^{21}$ M. Ben Amar, Phys. Rev. A 44, 3673 (1991).

${ }^{22}$ M. Ben Amar, Phys. Rev. A 43, 5724 (1991).

${ }^{23}$ R. C. Ball and R. M. Brady, J. Phys. A 18, L809 (1985).

${ }^{24}$ E. Somfai, N. Goold, R. C. Ball, J. P. DeVita, and L. M. Sander, Phys. Rev. E 70, 051403 (2004).

${ }^{25}$ R. C. Ball, N. E. Bowler, L. M. Sander, and E. Somfai, Phys. Rev. E 66, 026109 (2002).

${ }^{26}$ L. Paterson, Phys. Rev. Lett. 52, 1621 (1984).

${ }^{27}$ E. Brener, H. Levine, and Y. H. Tu, Phys. Rev. Lett. 66, 1978 (1991).

${ }^{28} \mathrm{H}$. Levine and Y. H. Tu, Phys. Rev. A 45, 1053 (1992).

${ }^{29}$ V. A. Bogoyavlenskiy, Phys. Rev. E 64, 066303 (2001). 